\title{
Changes in unpunished responding during response-contingent punishment
}

\author{
PHILIP J. DUNHAM \\ Dalhousie University, Halifax, Nova Scotia, Canada B3H 4JI
}

\begin{abstract}
In two experiments, gerbils that were punished for eating exhibited an increase in only the most probable of several ongoing alternative responses (running) during sessions when eating was suppressed. These results support previously suggested punishment and implicit avoidance rules for predicting the effects of response-contingent punishment in multiple-response baseline procedures. Most subjects also exhibited a decline in unpunished digging responses during punishment sessions. Neither this decline in the digging behavior nor the amount of increase in running behavior supported a quantitative constant proportion rule which predicts that each of the unpunished responses will increase to occupy a constant proportion of the time available to it.
\end{abstract}

Given unconstrained access to several types of activity in a relatively constant environment, most organisms will eventually exhibit some stable repertoire of measurable responses. Once responding has stabilized, it is possible to introduce any number of experimental manipulations and observe the adjustments which the organism makes in response to a particular manipulation.

Experimental procedures which approximate the above described situation are called multiple response baseline procedures (Dunham, 1971), and there is an increasing interest in studying the effects of various operations in a multiple response context (Bernstein \& Ebbesen, 1977; Dunham, 1971, 1977; Mazur, 1975; Shettleworth, 1972, 1973, 1975; Timberlake \& Allison, 1974).

In our laboratory, we have been primarily concerned with measuring the effects of various punishment operations introduced into multiple response situations. In an initial paradigmatic experiment with response-contingent punishment (Dunham, 1971), hungry male gerbils were permitted free access to paper for shredding, food pellets, and a drinking tube during daily experimental sessions. Response duration measures revealed preferences for each of these activities in the aforementioned order. Following the establishment of baseline behaviors, the animals were split into three groups in which paper shredding, eating, and drinking, respectively, were punished with response-contingent electric shock.

The results obtained in all three groups of this experiment yielded two consistent generalizations.

This research was supported by Project Grant APA-194 from the National Research Council of Canada. My thanks are extended to Carol Anderson and Susan Cohen for assisting in the collection and analysis of these data.
First, contrary to suggestions that many speciestypical behaviors are refractory to the effects of punishment (cf. Melvin \& Ervey, 1973; Walters \& Glazer, 1971), the punished response was effectively suppressed in all three groups. Second, and of more interest, the most probable of the remaining unpunished responses increased in duration during the punishment sessions. These two generalizations were referred to as the punishment rule and the implicit avoidance rule, respectively.

In a subsequent series of experiments, designed primarily to investigate the implicit avoidance rule, we used a two-response baseline procedure (Dunham, 1972). Thirsty rats were given free access to a running wheel and a drinking tube during daily experimental sessions. After baselines were established, the animals were punished for drinking. As the punishment and implicit avoidance rules mentioned above suggest, drinking was suppressed and an increase in running was observed during the punishment sessions. These data also suggested a rather precise quantitative rule for predicting the amount of increase which will be observed in unpunished alternative behavior. The rule can be stated simply as follows: Given two incompatible behaviors, each behavior will occupy a constant proportion of the time available to it. If, with running and drinking concurrently available, a rat spends $10 \%$ of its time running and $20 \%$ of its time drinking, it is spending $1 / 8$ of the time available for running in the state of running. If we eliminate access to the drinking tube, the constant proportion rule predicts that running will increase by $1 / 8$ of the additional time made available by the absence of the drinking response.

In two additional experiments, when access to either a running wheel or a drinking tube was simply blocked following baseline training, an increase in 
the unblocked response was observed, and again, the amount of increase was predicted very precisely by the constant proportion rule.

\section{EXPERIMENT 1}

The purpose of the first experiment in the present series was to answer two additional questions relevant to research in this area. First, will the punishment and implicit avoidance rules suggested by our initial multiple response baseline experiments with gerbils generalize to a different multiple response hierarchy? Specifically, will response-contingent punishment suppress the referent response and produce an increase in the most probable of the unpunished alternative responses? Second, how will the constant proportion rule generalize to a different multiple response hierarchy which contains more than two measurable responses? To date, this quantitative rule has been applied only in the context of a two-response (running and drinking) procedure. If the rule is tested in the context of a three- or fourresponse baseline, a number of questions can be answered. First, and most obvious, is whether the rule will continue to make accurate predictions in the context of the new response hierarchy. Second, consider two different ways in which the constant proportion rule can be applied in the context of baselines with three or more responses. One can suggest that the rule will apply to only the most probable of the unpunished responses in the response repertoire. As such, the constant proportion rule is simply a more precise, quantitative version of the qualitative implicit avoidance rule. Alternatively, one can suggest that each of the unpunished responses will increase to occupy a constant proportion of the time made available by the absence of the punished response. Which, if either, of these approaches is correct?

To answer these two questions, a multiple response environment for gerbils was designed to permit the measurement of a four-response hierarchy not previously investigated.

\section{Method}

Subjects. Six mature, male Mongolian gerbils (Meriones uniguiculatus) between 70 and $80 \mathrm{~g}$ were obtained from Tumblebrook Farm, Brant Lake, New York, to serve as subjects. The animals were housed throughout the experiment in individual plastic cages with sawdust bedding.

Apparatus. The apparatus was a wooden chamber $43 \mathrm{~cm}$ wide $\times 30 \mathrm{~cm}$ deep $\times 28 \mathrm{~cm}$ high. The grid floor, constructed of $3 . \mathrm{mm}$ stainless steel rods spaced $1 \mathrm{~cm}$ apart, permitted the delivery of scrambled electric shock from a Grason-Stadler Model 700 shock generator. The front wall of the chamber was made of Plexiglas for observation purposes and also served as a door. Centered on this wall was a food bin which permitted continuous access to sunflower seeds through a $2 \times 4 \mathrm{~cm}$ opening in the wall. The rear wall of the chamber permitted access to the lower half of a small activity wheel, with an $11-\mathrm{mm}$ radius, mounted on a Wahmann activity wheel hub. The running surface of the wheel was lined with fine grit sandpaper to facilitate traction. An open rectangular box $(13 \times 30 \times 11 \mathrm{~cm})$ was affixed to one side wall of the cham ber. The box was filled to a depth of $5 \mathrm{~cm}$ with washed builder's sand. Finally, a slot was centrally located $6 \mathrm{~cm}$ above the floor on the other side wall of the chamber through which a piece of bristol board $(5 \times 15 \mathrm{~cm})$ could be inserted and fixed in position. A small rectangular board $(5 \times 10 \mathrm{~cm})$ was located under the slot 10 provide a platform on which the gerbils could stand while shredding the bristol board. A translucent Plexiglas ceiling with a $7.5-\mathrm{W}$ white bulb above it illuminated the chamber during experimental sessions.

The entire chamber was enclosed in a large sound-insulated box with a Plexiglas window for observation and a fan to provide masking noise and ventilation. An observer was seated in the dark outside this box and provided with a four-switch keyboard for recording the duration of each of the four activities. Depressing a switch activated the appropriate duration timers and counters for each response. Devices for recording these data were located in an adjacent room.

Procedure. Upon arrival, the animals were maintained in the laboratory in continuous light at an ambient temperature of $20^{\circ} \mathrm{C}$ with ad-lib access to laboratory chow and water for 2 weeks. During the second week of adaptation, daily food intake was monitored, and for 5 days immediately prior to the start of the experiment, each gerbil was restricted to $80 \%$ of its normal daily food intake and $30 \mathrm{~min}$ access to water.

Following 5 days on this deprivation schedule, the animals were given daily 30-min baseline sessions in the multiple response chamber for 10 consecutive days. During each session, each animal had free access to an unlimited supply of sunflower seeds, a $5 \times 15 \mathrm{~cm}$ piece of bristol board, a running wheel, and the box of sand. On Days 7,9 , and 10 , an observer recorded the amount of time the animal spent in each of these four activities.

Following the 10 baseline sessions, a punishment contingency was introduced whereby a scrambled .4-mA .5-sec electric shock was delivered via the grid floor simultaneous with the onset of the eating response. The shock was continued at 2 -sec intervals if the animal persisted in making the response after the initial shock. The punishment procedure continued for 7 consecutive 30 -min sessions during which the observer recorded the duration of each of the four behaviors. Following 7 days of punishment training, the shock contingency was eliminated and the animals were observed for 2 days of recovery.

Throughout the experiment, the four behaviors of interest were operationally defined as follows: (1) paper shredding onset and of fset were defined as the initiation and the termination of any oral contact with the bristol board which was accompanied by chewing movements; (2) the onset and offset of running behavior were defined as the initiation and termination of continuous wheel rotation (this does not include minor rocking movements sometimes observed); (3) the onset and offset of eating behavior were defined as the initiation of oral contact with the food and the termination of chewing movements (note that the animals usually consumed several seeds with each approach to the food hopper, defining "bouts" of eating without a "measurable" interval between successive seeds); and (4) the onset and offset of scratching in the dirt were defined simply as entry and departure from the sand box (a variety of different behaviors were actually observed while the animals were in the sand, with digging and scratching movements the most frequent).

It should also be noted that records were kept of the number of sunflower seeds consumed during each daily session. During punishment training, when eating behavior was suppressed, each animal was given its average baseline ration of sunflower seeds in a supplemental home feeding cage $1 \mathrm{~h}$ after the daily experimental session. Hence, the introduction of the punishment contingency was not confounded with an increase in deprivation levels during punishment sessions. 


\section{Results and Discussion}

Using the response duration measure, the results obtained for each subject during each phase of the procedure are presented in Figure 1. During baseline training, the response duration measure revealed that all subjects preferred running over the other behaviors, followed by dirt and eating, in that order. The one minor exception to this consistent response hierarchy was a reversal of the eating and dirt responses seen in Subject 5.

Note also that we did not observe any measurable amount of paper shredding during the baseline phase of the experiment in any of the subjects. This contrasts sharply with results obtained in other multiple response situations where paper shredding is typically a highly preferred activity for these animals (Dunham, 1971). Apparently, the availability of the running wheel and/or dirt is sufficient to inhibit the shredding activity (cf. Dunham, 1977).

Consider next the effects of the punishment contingency upon both punished and unpunished responses. As expected, the punishment contingency suppressed eating behavior in all subjects. It should also be noted that grid-biting behavior, often associated with the introduction of shock in earlier studies, was not observed in this apparatus.

Of more interest were the changes in unpunished baseline responses. All subjects, except Subject 1, revealed two consistent changes in unpunished responding: (1) a substantial increase in the total duration of running behavior developed during punishment training, and (2) a gradual decline in the amount of time spent in the dirt. As noted above, Subject 1 deviated from these generalizations and revealed an increase in paper shredding during punishment, while both running and the dirt responses declined. In general, these data provide additional support for the qualitative punishment and implicit avoidance rules derived from earlier research using a different response hierarchy: (1) There was an immediate decline in the duration of the punished response, eating; and (2) the most probably of the remaining unpunished alternative responses, running, increased in total duration.

Consider next the quantitative predictions made by the constant proportion rule. Recall that this rule emerged from research using a two-response (running and drinking) baseline (Dunham, 1972). If, for the moment, we consider only the response which increased in duration (running) and the punished response (eating) in our analysis, and consider only the asymptotic values (last 3 days of punishment training), the observed and predicted increases in running are presented in Table 1 . As the data for the five gerbils that exhibited an increase in running during punishment indicate, there is considerable discrepancy between observed and predicted values, with the exception of Subject 4.

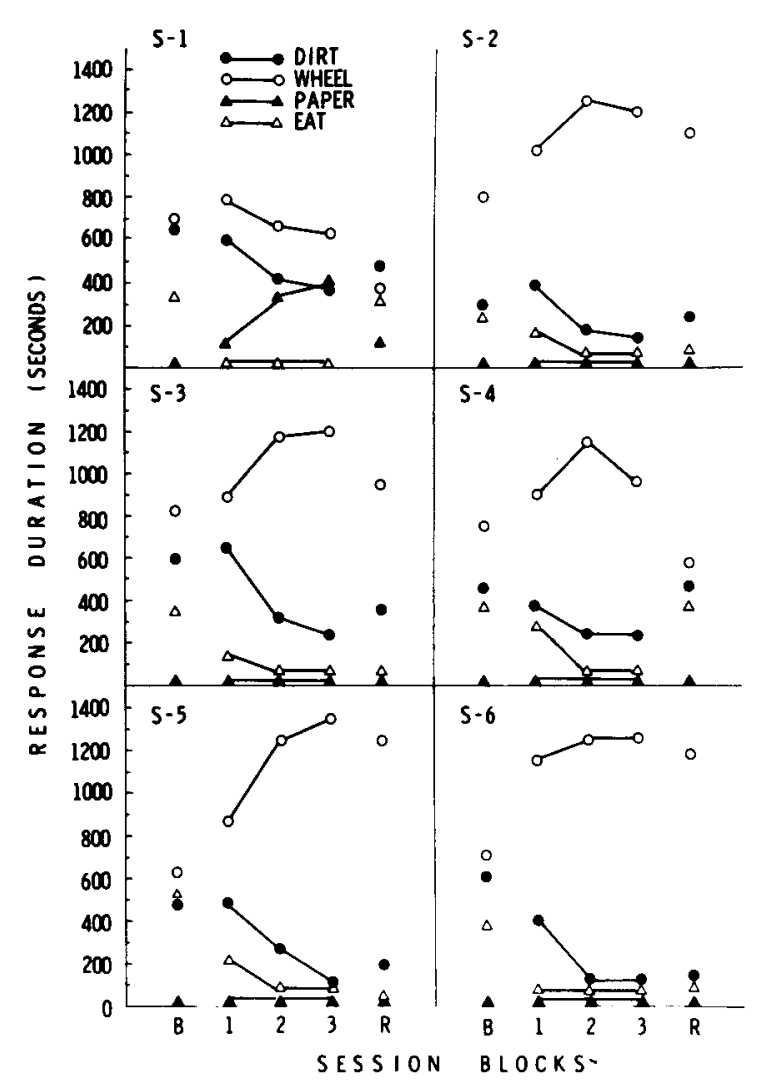

Figure 1. Total duration of each response for each of the six subjects during all three phases of the procedure. Baseline data are plotted as a three-session block; punishment data as two two-session blocks and a final three-session block; and recovery data as a two-session block.

If we now consider all of the responses in this procedure, it is also clear that the decline in the dirt response during punishment sessions also questions the validity of the constant proportion rule. As stated earlier, one potential implication of the constant proportion rule, when extended to baseline procedures including more than two responses, is that each of the unpunished alternative responses will increase in duration when the punished response is eliminated from the repertoire. Hence, the decline in the amount of time spent in the dirt also questions this implication of the quantitative rule.

Asymptotic Value of Running Predicted by the Constant Proportion Rule and Values Actually Observed in Five Subjects that Revealed an Increase in Running During Punishment for Eating

\begin{tabular}{crr}
\hline & \multicolumn{2}{c}{ Duration (in Seconds) } \\
\cline { 2 - 3 } Subject & Predicted & Observed \\
\hline 2 & 945.6 & $1,215.7$ \\
3 & $1,081.7$ & $1,206.6$ \\
4 & 968.3 & 967.0 \\
5 & 845.8 & $1,307.0$ \\
6 & 934.3 & $1,255.0$ \\
\hline
\end{tabular}


The recovery data presented in Figure 1 are somewhat inconsistent. In cases where eating did recover its baseline strength within the two recovery sessions (e.g., Subjects 1 and 4), other responses also tended to return to baseline. In other subjects, no recovery of eating was observed and the trends observed in unpunished responses during punishment also persisted.

A measure of the frequency of response initiations during punishment training revealed results similar to the duration measure presented in Figure 1. The running response increased above baseline levels, while eating and dirt responses declined. There was, however, a gradual decline in the frequency of running during the course of punishment training. When compared to the increase in the duration of running during punishment seen in Figure 1, these former data indicate that the animal is adjusting its behavior by running for longer bouts less frequently as punishment training progresses.

Taken together, the data obtained in the present experiment provide reasonably definitive answers to the questions posed in the rationale for the experiment. First, in this novel response hierarchy, responsecontingent punishment suppressed the punished response and produced an increase in the most probable of the unpunished alternative responses. As such, these data provide additional support for the punishment and implicit avoidance rules. Apparently, these generalizations are not specific to the response hierarchy under consideration.

Second, the data seriously question the generality of the quantitative constant proportion rule. Neither the amount of increase in running nor the decline in dirt responses are predicted by this rule as presently formulated.

\section{EXPERIMENT 2}

Recall that in the first experiment the amount of time spent in the dirt declined in all six subjects during the punishment sessions. This decline runs exactly opposite to the predictions made by the constant proportion rule and is not subsumed by either the punishment or implicit avoidance rule. The latter, as presently formulated, makes reference only to changes in the punished response and the most probable of the unpunished alternatives. This leaves one with the problem of interpreting, and consequently predicting, changes like the decline in dirt responses during punishment.

Perhaps the most parsimonious interpretation of the decline in dirt responses is to view it as a response competition phenomenon. Specifically, the sizable increases in unpunished running may compete for available time with the other, less probable, unpunished alternative responses. One might even entertain notions about a quantitative rule based upon the relative durations of the unpunished responses to predict the amount of decline in each unpunished response.

The second experiment in this series was a systematic replication of Experiment 1 with two changes in procedure designed to test some implications of the response competition notion described above. Specifically, if the increase in running behavior which has been observed in this context is competing for available experimental time with all other less probable unpunished responses in the repertoire, any unpunished behavior we observe in addition to the dirt responses (e.g., paper shredding) should also decline in duration during punishment sessions. To this end, two changes in procedure were instituted in an attempt to generate some unpunished paper shredding behavior during baseline and punishment sessions. First, the subjects were provided with bristol board in their home cages for 5 days prior to the start of the experiment in an attempt to "prime" this response. Second, the length of each experimental session was increased from 30 to $40 \mathrm{~min}$ to provide extra time for any shredding behavior to occur.

\section{Method}

Subjects and Apparatus. Six additional experimentally naive gerbils from the same population as those used in Experiment 1 were used in the present experiment.

Procedure. All aspects of the procedure were identical to those described in Experiment I with the exceptions noted in the introduction.

\section{Results and Discussion}

Figure 2 presents the response duration measures for each of the six subjects in Experiment 2 for each phase of the experiment. Three points should be noted with reference to the individual data. First, the paper shredding behavior was of primary interest in the present experiment. In spite of efforts to "prime" this response, only one subject (Subject 5) exhibited any paper shredding during the baseline phase of the procedure. As the data from this subject indicate, the baseline durations of this response were maintained throughout the punishment phase of the procedure. Note also that each of the other five subjects exhibited very small amounts of paper shredding which developed during punishment sessions. As such, these results provide only weak evidence against the response competition notion as an explanation of the decline in dirt responses. Further research with a four-response repertoire will be necessary to settle this question.

A second point concerns some of the inconsistency in the individual data from this second experiment. Note that 2 subjects ( 2 and 5) actually spent more time in the dirt during punishment sessions. While it is not clear why these 2 subjects differed in this 


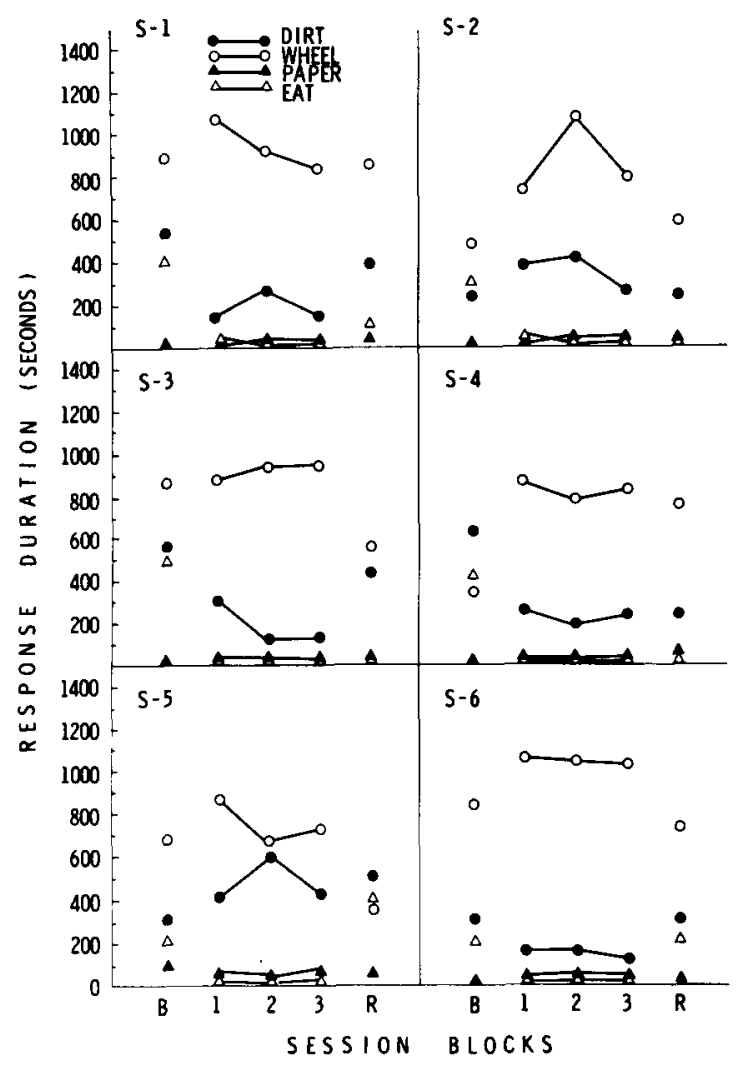

Figure 2. Total duration of each response for each of the six subjects during all three phases of the procedure. Session data blocked as in Figure 1.

respect from the other 10 subjects in these experiments, it can be argued that these deviations also run counter to the response competition notion. If both dirt responses and running responses can increase in duration, it does not seem likely that the two are competing for available experimental time.

Finally, it should be noted that the response frequency measures in this second experiment lead to essentially the same conclusions as discussed in Experiment 1.

To summarize briefly, the results of the second experiment essentially replicate those obtained in the first experiment and provide some evidence against a simple response competition interpretation of the decline in unpunished dirt responses.

\section{GENERAL DISCUSSION}

On the basis of previous research (Dunham, 1971, 1972), I tentatively outlined several empirical generalizations intended to predict the effects of various punishment operations in multiple response environments. With respect to response-contingent punishment, three testable rules were proposed: (1) a punishment rule, which states that the referent, or punished, response will be suppressed relative to its baseline duration; (2) an implicit avoidance rule, which states that the most probable of the unpunished alternative responses will increase in duration relative to its baseline value; and (3) a constant proportion rule, which makes quantitative predictions concerning the amount of increase in unpunished behavior. Data from the two experiments in the present series suggest three points of information relevant to the above generalizations, each of which deserve further discussion.

First, consider the support for the punishment and implicit avoidance rules. In the past decade, a wide variety of theory and research with animals has stressed the importance of certain biological constraints the organism brings into an experimental situation. In discussing aversive conditioning procedures, Bolles (1970), Melvin and Ervey (1973), Shettleworth (1972) and Walters and Glazer (1971), among others, have suggested that it is necessary to consider the functional relevance of certain speciestypical behaviors if we wish to predict the effects of aversive stimulation on these patterns of behavior. In general, these positions maintain that certain categories of behavior (e.g., defensive behaviors) may be refractory to the effects of punishment contingencies and particularly responsive to manipulation by avoidance contingencies. Other categories of behavior are considered uniquely susceptible to disruption by punishment and not particularly effective as avoidance responses.

The data from our past research and the present experiments question the generality of these arguments. In experiments we have conducted thus far, the two empirically derived rules called the punishment rule and the implicit avoidance rule have predicted the changes in behavior produced by responsecontingent punishment. The punished response has declined in duration, and the most probable unpunished alternative has increased in duration without respect for any post hoc claims about the functional relevance of the particular responses involved. Considering these data, and some of the methodological problems associated with data supporting the functional relevance notion (cf. Dunham, 1977), it is suggested that the inductively generated punishment and implicit avoidance rules are a viable approach to predicting the effects of punishment procedures in the context of multiple response baseline procedures.

A second point of discussion concerns the decline in the duration of unpunished dirt responses observed in these two experiments. Since the data in Experiment 2 contraindicate a response competition interpretation, and the dirt response is not subsumed by the predictions of either the punishment or implicit avoidance rules, one is left with the problem of predicting changes in responding for unpunished responses other than the most probable response in the repertoire. Several approaches to this problem are possible. One might, for example, revert at this 
point to a biological constraints approach and discuss the functional relevance of various unpunished responses. The dirt responses in question make the point that it is often difficult to specify a priori the functional relevance of a particular response and the impact which painful stimulation might have upon the response. Should we, for example, consider burrowing behavior a part of the gerbil's defense repertoire, a nesting behavior, or perhaps foodseeking behavior?

An alternative approach is to search through the descriptive data available on the multiple response repertoire to determine if the dirt response has any unique orderly properties upon which to base future predictions about changes in this and other unpunished responses. It is exactly this inductive, empirical approach which generated the punishment and implicit avoidance rules tested in the present research. One such factor which is potentially important in multiple response procedures, and which has not received any attention thus far, is the sequential dependencies which can exist between unconstrained responses in the baseline condition. Although we do not have a quantitative analysis available, informal observations of the gerbils in the present research suggest the possibility of a strong sequential dependency between eating and dirt responses in that order. Such sequential dependencies may provide a basis for predicting changes like the decline in the dirt response when eating is suppressed; or increases in unpunished alternatives other than the most probable response in the repertoire. We are, at present, attempting to examine the potential role of such sequential dependencies in predicting adjustments to response-contingent shock in the multiple-response context.

A third point of discussion concerns the failure of the present research to provide support for the quantitative constant proportion rule. The attempt to make quantitative predictions across species and responses is clearly premature. It should also be noted that the implications of these negative data go beyond the punishment procedures we have considered. Some recent data reported by Mazur (1975) can also be related to the constant proportion rule. In Mazur's experiment, thirsty rats were given free access to a running wheel and drinking tube during daily experimental sessions. After stable baseline behaviors were established, a somewhat unique interdependent contingency operation was introduced. The interdependent contingency required the rat to run for some fixed period of time in order to gain access to the drinking tube. Once the tube was presented, the wheel was locked, and the animal was required to drink for some fixed period of time to regain access to the wheel, and so on.
Using this type of interdependent contingency, Mazur systematically varied the proportion of time the rat was required to drink (or run) in the run-plusdrink interdependent condition. For example, an interdependent contingency which required $20 \mathrm{sec}$ of running for $5 \mathrm{sec}$ of drinking defined a proportion of required drinking (p) of .2 and a proportion for required running $(1-p)$ of .8 .

In the context of this procedure, Mazur proposed a quantitative model designed to predict the changes in running and drinking (relative to baseline values) that would be produced by interdependent contingencies for all possible values of $\mathrm{p}$. This model, which finds its heritage in Herrnstein's $(1961,1970)$ matching law and Premack's (1971) differential probability principles, fit the data Mazur obtained with various running and drinking contingencies very well.

We need not consider the details of Mazur's quantitative model in the present context, except to note two important points. First, an interdependent contingency in which $p$ has a value of zero indicates essentially that the experimenter has completely blocked access to one of the responses and permitted free access to the alternative response. Hence, this type of contingency is functionally equivalent to the operations used in the experiments with running and drinking reported by Dunham (1972) and described above. Second, when $p$ is at the limiting value of zero, Mazur's equations for predicting the effects of blocking access to one response are essentially reducible to the constant proportion rule discussed earlier. Hence, both Mazur's model and the constant proportion rule make identical predictions when, following multiple response baseline training, access to one behavior is blocked or suppressed by a punishment operation. Consequently, the failure of the data in the present experiment to support the constant proportion rule also questions the generality of the assumptions of Mazur's model. Both fail to predict the amount of increase in running and the decline in dirt responses observed in the present research.

Finally, it should be noted that the focus of the present research has been on the generality of these empirically derived rules, not upon the mechanisms which might underlie the changes in behavior produced by the response-contingent punishment procedure. I have discussed the implications of these rules for traditional punishment theory in other contexts (cf. Dunham, 1971; Dunham \& Carr, 1976). In view of the fashionable contemporary trend toward arguing against the generality of empirical generalizations across species and responses, we have given first priority to considering the generality of these rules prior to elaborating further on the mechanisms which might underlie the rules. 


\section{REFERENCES}

Bernstein, D. J., \& EBbesen, E. B. Interaction of structure and function in the stream of human behavior. Joumal of Experimental Psychology: General, 1977, in press.

Bolles, R. C. Species-specific defense reactions and avoidance learning. Psychological Review, 1970, 77, 32-48.

Dunham, P. J. Punishment: Method and theory. Psychological Review, 1971, 78, 58-70.

Dunham, P. J. Some effects of punishment upon unpunished responding. Joumal of the Experimental Analysis of Behavior, $1972,17,443-450$.

Dunham, P. J. The nature of reinforcing stimuli. In V. Honig \& J. Staddon (Eds.), Handbook of operant behavior. Englewood Cliffs, N.J: Prentice-Hall, 1977.

Dunham, P. J., \& CarR, A. Pain-elicited aggression in the squirrel monkey: An implicit avoidance contingency. Animal Learming \& Behavior, 1976, 4, 89-95.

HeRrNSTEIN, R. J. Relative and absolute strength of response as a function of frequency of reinforcement. Joumal of the Experimental Analysis of Behavior, 1961, 4, 267-272.

HeRRnsteIn, R. J. On the law of effect. Journal of the Experimental Analysis of Behavior, 1970, 13, 243-266.

MAzUR, J. E. The matching law and quantifications related to Premack's principle. Journal of Experimental Psychology: Animal Behavior Processes, 1975, 1, 374-386.

Melvin, K., \& ERvey, D. Facilitative and suppressive effects of punishment of species-typical aggressive display in Betta splendens. Journal of Comparative and Physiological Psychology, 1973, 83, 451-457.

Premack, D. Catching up with common sense or two sides of a generalization: Reinforcement and punishment. In R. Glazer (Ed.), The nature of reinforcement. New York: Academic Press, 1971

Shettlew ORTh, S. J. Constraints on learning. In D. S. Lehrman, R. A. Hinde, \& E. Shaw (Eds.), Advances in the study of behavior (Vol. 4). New York: Academic Press, 1972.

Shettleworth, S. J. Food reinforcement and the organization of behavior in golden hamsters. In R. Hinde \& J. Hinde (Eds.), Constraints on learning. New York: Academic Press, 1973.

SheTtleworth, S. J. Reinforcement and the organization of behavior in golden hamsters: Hunger, environment, and food reinforcement. Journal of Experimental Psychology: Animal Bchavicr Procasses, 1975, 1, 56-87.

Timberlake, W., \& Allison, J. Response deprivation: An empirical approach to instrumental performance. Psychological Review, 1974, 81, 146-164.

Walters, G. C., \& Glazer, R. D. Punishment of instinctive behavior in the Mongolian gerbil. Journal of Comparative and Physiological Psychology, 1971, 75, 331-340.

(Received for publication June 14, 1977; revision accepted September 30, 1977.) 